\title{
Orthopédie-Traumatologie Une nouvelle revue d'Orthopédie-Traumatologie, pour quoi faire?
}

La S.O.T.EST (Société d'OrthopédieTraumatologie de l'Est de la France) et le GECO (Groupe d'Etude pour la Chirurgie Osseuse) viennent de s'unir pour co-éditer, sous la bannière de SpringerVerlag France une nouvelle revue d'orthopédie-traumatologie à parution trimestrielle, dont voici le premier numéro. Cette nouvelle revue a plusieurs ambitions; permettre aux deux sociétés fondatrices de publier des travaux référencés dans les grandes bibliographies internationales, permettre au plus grand nombre de jeunes chirurgiens, en particulier chefs de clinique - assistants, pour les français, de voir publiés leurs travaux, les revues nationales étant encombrées et saturées, s'ouvrir au dialogue européen, à l'aube d'une Europe qui, si elle a du mal à trouver sa dimension politique, existe déjà, sans aucune limitation, dans les idées et les sciences.

La S.O.T.EST, née en 1973, réunit environ 250 membres, essentiellement orthopédistes et traumatologues, mais aussi chirurgiens de la main, rhumatologues, rééducateurs, anesthésistes, des cinq régions du "Grand Est", à savoir l'Alsace, la Bourgogne, la ChampagneArdennes, la Franche-Comté et la Lorraine, mais aussi d'Allemagne, de Bel- gique, d'Italie, du Luxembourg et de Suisse. Elle organise chaque année une grande réunion, avec en règle, deux thèmes principaux et des communications libres, dans un de ses nombreux centres urbains, tantôt en milieu universitaire, tantôt en non universitaire, public ou privé. Depuis quatre ans, ont eu lieu deux rencontres franco-allemandes très enrichissantes. Auparavant avaient déjà eu lieu des rencontres franco-belge et franco-suisse très enrichissantes.

Le GECO est né en 1976 avec comme idée directrice d'offrir une plateforme de rencontres annuelles sur des thèmes orthopédiques et traumatologiques, en ambiance de ski hivernal, où, en conjuguant activités sportives et travail, peuvent se rencontrer hors de tout formalisme des chirurgiens orthopédistes et traumatologues de tous horizons, universitaires et non universitaires. Les membres fondateurs et titulaires sont d'accord pour chaque année, mettre leurs dossiers en commun pour aboutir à une table ronde, publiée ensuite sous forme de rapports. Plus de 20 rapports ont été publiés. Les séances du GECO sont, bien entendu ouvertes à d'autres participants que les seuls membres titulaires, et constituent chaque année en janvier un must pour de nombreux habitués. En janvier 1991 a eu lieu la XV ème Réunion Elargie avec 160 participants chirurgiens. Depuis deux ans, les réunions du GECO sont bilingues, en français avec interprétation en anglais.

L'origine européenne des membres de la S.O.T.EST, la position centrale, lotharingienne, de la grande région de l'est française, la volonté d'ouverture européenne du GECO d'autre part, nous ont poussé à vouloir une revue "européenne", concrétisée par une parution d'emblée en français et en anglais avec résumé dans chaque langue. L'on ne s'intitule pas européen, on l'est réellement ou non. Conscients de l'être quotidiennement de par notre géographie et notre culture, nous souhaitons apporter par cette nouvelle revue une brique solide à l'édifice nouveau en cours de réalisation dans notre vieux continent.

Professeur Pierre Kehr

Professeur Henry Coudane

Rédacteurs en Chef 